\title{
A Study to Decipher the Potential Effects of Butylphthalide against Central Nervous System Diseases Based on Network Pharmacology and Molecular Docking Integration Strategy
}

\author{
Qinqin Zhao $\mathbb{D}^{1,2}$ Bei Zheng $\mathbb{D}^{1},{ }^{1}$ Pinpin Feng $\mathbb{D}^{1},{ }^{1}$ and Xiang $\mathrm{Li} \mathbb{C}^{3}$ \\ ${ }^{1}$ Department of Pharmacy, Tongde Hospital of Zhejiang Province, Hangzhou, Zhejiang Province, China \\ ${ }^{2}$ Zhejiang Academy of Traditional Chinese Medicine, Hangzhou, Zhejiang Province, China \\ ${ }^{3}$ School of Basic Medical Sciences and Forensic Medicine, Hangzhou Medical College, Hangzhou, Zhejiang, China
}

Correspondence should be addressed to Xiang Li; leexiang2004@126.com

Received 15 December 2020; Revised 5 April 2021; Accepted 27 April 2021; Published 5 May 2021

Academic Editor: Woon-Man Kung

Copyright (c) 2021 Qinqin Zhao et al. This is an open access article distributed under the Creative Commons Attribution License, which permits unrestricted use, distribution, and reproduction in any medium, provided the original work is properly cited.

Background. Butylphthalide (NBP), approved by the China National Medical Products Administration (NMPA) for the treatment of ischemic stroke (IS), showed pleiotropic potentials against central nervous system (CNS) diseases, including neuroprotection and cognitive deficits improvement. However, the effects and corresponding modes of action were not fully explored. This study was designed to investigate the potential of NBP against IS-associated CNS diseases based on network pharmacology (NP) and molecular docking (MD). Methods. IS was inputted as the index disease to retrieve the "associated diseases" in DisGeNET. Threedatabase-based IS genes were obtained and integrated (DisGeNET, Malacards, and OMIM). Then, IS-associated genes were identified by combining these genes. Meanwhile, PubMed references and online databases were applied to identify NBP target genes. The IS-related disease-disease association (DDA) network and NBP-disease regulation network were constructed and analyzed in Cytoscape. In silico MD and references were used to validate the binding affinity of NBP with critical targets and the potential of NBP against certain IS-related CNS disease regulation. Results. 175 NBP target genes were obtained, while 312 ISrelated disease genes were identified. $36 \mathrm{NBP}$ target genes were predicted to be associated with IS-related CNS diseases, including Alzheimer's disease (AD), epilepsy, major depressive disorder (MDD), amyotrophic lateral sclerosis (ALS), and dementia. Six target genes (i.e., GRIN1, PTGIS, PTGES, ADRA1A, CDK5, and SULT1E1) indicating disease specificity index (DSI) $>0.5$ showed certain to good degree binding affinity with NBP, ranging from -9.2 to $-6.7 \mathrm{kcal} / \mathrm{mol}$. And the binding modes may be mainly related to hydrogen bonds and hydrophobic "bonds." Further literature validations inferred that these critical NBP targets had a tight association with AD, epilepsy, ALS, and depression. Conclusions. Our study proposed a drug-target-disease integrated method to predict the drug repurposing potentials to associated diseases by application of NP and MD, which could be an attractive alternative to facilitate the development of CNS disease therapies. NBP may be promising and showed potentials to be repurposed for treatments for $\mathrm{AD}$, epilepsy, ALS, and depression, and further investigations are warranted to be carefully designed and conducted.

\section{Introduction}

Brain/central nervous system (CNS) diseases are multifactorial and polygenic diseases, including stroke, Alzheimer's disease (AD), and epilepsy, which remain an urgent and unmet medical need nowadays. The WHO statistics titled "Global Health Estimates 2016: Estimated deaths by age, sex, and cause" showed that there were
56873805 deaths globally in which noncommunicable diseases (NCDs) contributed $71.29 \%$ (40545176 deaths). Currently, NCDs are the main reasons to cause deaths, and ischemic heart disease (IHD) and stroke are ranked $1^{\text {st }}$ and $2^{\text {nd }}$, respectively, among the top 10 causes of death. The data indicated that brain/CNS-related NCDs of stroke, neurological conditions, and brain/nervous system cancers contributed to $15 \%$ of deaths. Meanwhile, the 
proportions of IHD and stroke are $16.59 \%$ and $10.16 \%$, respectively.

It has a high failure rate to develop therapies for brain/ CNS-related NCDs, as AD, epilepsy, Parkinson's disease, and dementia, which will be costly, lengthy, and characterized by poor/no understanding of the underlying pathophysiology [1]. New strategies to develop drugs for CNS diseases treatments are urgently needed. Fortunately, multiple CNS-disease-related pathways have been identified, and CNS diseases were found to share common pathways, which will provide possible opportunities for applying existing drugs for other indications based on disease-disease associations (DDAs) [2]. Diseases with overlapping disease modules display similar underlying molecular mechanisms and clinical symptoms [3], which will favor us to find approved drugs of one indication to other indications based on DDAs.

As de novo discovering and developing new drugs are extremely tough works with time-consuming, high-cost, and low-success rate. For CNS drug development, it is particularly tough as researchers need to conquer the hurdles encountered, like the blood-brain barrier (BBB) and the translations from animal to human, etc. [4]. Drug repurposing or drug repositioning would be an attractive way to develop therapies for CNS diseases. While de novo drug development and approval require at least $12-16$ years and 1-2 billion dollars, drug repositioning only needs 6 years and 300 million dollars. As most repurposed drugs have already passed the preclinical development and clinical testing, drug repositioning may facilitate the efficiency of drug development and it would be an important alternative to develop drugs for new indications [5]. Several examples have applied a drug repurposing strategy to accelerate drug development processes. Minocycline was repurposed for acute ischemic stroke (IS), as it showed effects of anti-inflammation and MMP-9 inhibition, which would be attractive to use in combination with tissue plasminogen activator (tPA) [6]. $\mathrm{AD}$ is a chronic and progressive neurodegenerative disease and one study reviewed priority candidate drugs for repositioning in $\mathrm{AD}$ [7], such as benperidol [8] and cinitapride [9]. Another study combining RNA sequencing and zebrafish model of seizures repurposed FDA-approved metformin, nifedipine, and pyrantel tartrate as candidate antiepilepsy drugs [10]. As dementia and type 2 diabetes (T2D) share some underlying pathophysiology, studies tried to repurpose T2D drugs for dementia treatment. Intranasal insulin, metformin, and GLP-1 receptor agonists are attractive candidate drugs; however, more studies are required before clinicians recommend candidate diabetes therapies for dementia [11]. Amyotrophic lateral sclerosis (ALS), as CNS rare disease, is a progressive fatal disorder. The free radical scavenger edaravone has been developed for the treatment of ALS [12]. In our previous study, we tried to find approved cardiovascular drugs for ischemic cerebrovascular disease based on disease-disease-associated prediction, which may provide a promising alternative to infer novel disease indications for known drugs [13]. DDAs link different diseases sharing common underlying molecular mechanisms together and provide beneficial information for drug repurposing investigation. What's more, the drug discovery paradigm has shifted from "magic bullet" to "multitarget drugs," as polypharmacology is the nature of most ligand/drugs, and the proportion of drugs that in theory could be repositioned is about $75[5,14,15]$. In 2002, dl-3n-butylphthalide (NBP) proved its safety and therapeutic effect on cerebral ischemia and received approval from the National Medical Products Administration (NMPA) of China for the treatment of acute ischemic stroke. NBP has shown potentials for treatment in the field of CNS diseases; several investigations have reported the effects of NBP including neuroprotection and cognitive deficits improvement [16-18]. In 2018, NBP was granted orphan drug designation by the US FDA for the treatment of ALS.

In order to repurpose NBP for the treatment of ISassociated CNS diseases and to decipher the potential underlying mechanisms, our study identified NBP potential targets by integration of targets from public databases like PubMed, PharmMapper [19], DRAR-CPI [20], and SymMap [21], while IS and other IS-associated CNS disease-related targets were obtained from DisGeNET [21], Malacards [22], and OMIM [23]. As mentioned above, CNS diseases may share common pathways, and disease-associated genes can be collected from disease databases (DisGeNET, Malacards, and OMIM). Network pharmacology and in silico molecular docking were integrated to uncover the underlying modes of action.

The schematic diagram can be attained in Figure 1 which depicted the three-layer drug-target-diseases network of NBP. In summary, our study has proposed a drug-targetdisease integrating method to predict the drug repurposing potentials to other associated diseases by application of network pharmacology and molecular docking, which could be an attractive alternative to facilitate CNS disease therapy development.

\section{Materials and Methods}

The network pharmacology and molecular modeling software used included AutoDock Tools (Version 1.5.6), AutoDock Vina software [24], LigPlot+ (Version 2.2), and Cytoscape 3.8.0 software [25].

\subsection{NBP Potential Target Identification and Integration.} NBP targets were collected and integrated from three resources, i.e., PubMed references, online database prediction (PharmMapper and DRAR-CPI), and SymMap. Firstly, butylphthalide was selected as a keyword to retrieve targets from PubMed by applying PALM-IST (https://www.hpppi. iicb.res.in/ctm/index.html) ranging from 2000 to 2014, while other targets from 2015 to $30^{\text {th }}$ May 2019 of NBP were obtained from PubMed with further reading and manually confirmation. Secondly, PharmMapper Server was applied to identify potential target candidates of NBP, and the .mol2 file was generated with ChemDraw and Chem3D software (version 17.1) according to the structure in PubChem (PubChem CID: 61361). In PharmMapper, the inclusion standard was Fit score $\geq 2$ with Human species. DRAR-CPI 


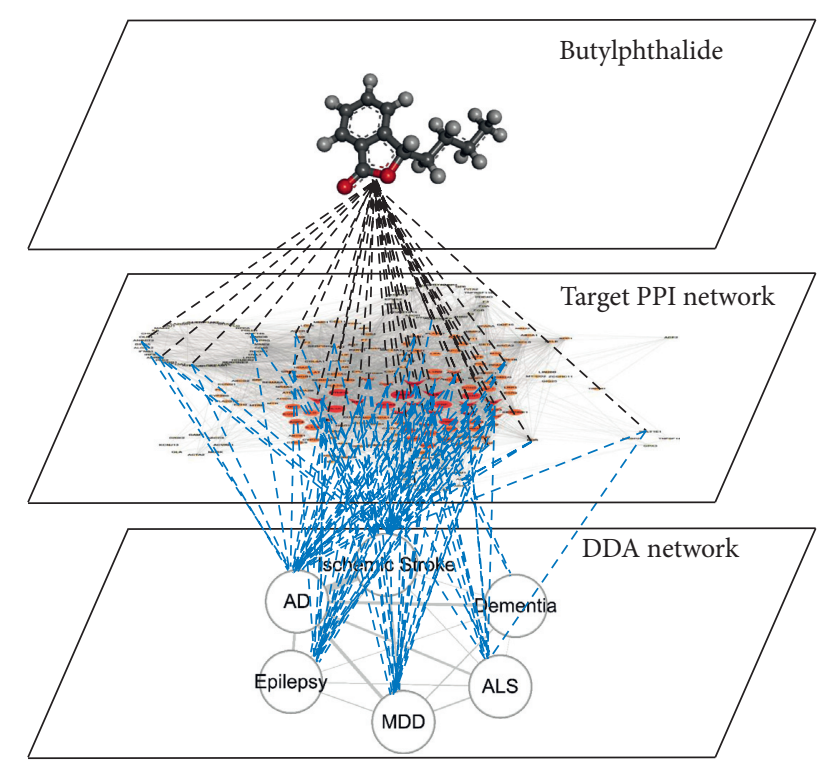

Figure 1: The three-layer drug-target-disease network of butylphthalide. PPI: protein-protein interaction; DDAs: diseasedisease associations; AD: Alzheimer's disease; MDD: major depressive disorder; ALS: amyotrophic lateral sclerosis. The thickness of edges in the DDA network is in proportion to the number of shared target genes in the target PPI network.

was another database applied to predict potential targets of NBP through inputting a .mol file from Drugbank (ID DB12749). In DRAR-CPI, the putative target selection standard was docking score $>-50$ and $Z^{\prime}$-score $>-0.5$. Targets from PharmMapper and DRAR-CPI were normalized, and their overlapped target genes were collected. Thirdly, SymMap database was used to retrieve potential targets of NBP with the keyword "Dl-3N-Butylphthalide." Duplicated NBP target candidates from different sources were removed, and the left target genes were used for further analysis.

2.2. IS-Related Disease-Disease Association (DDA) Network. DisGeNET database was applied to retrieve IS-related diseases and their corresponding genes. "Ischemic stroke" was used as index-disease (Index-disease-id: C0948008), and the "associated diseases" were obtained. According to the number of shared genes with IS, five CNS diseases were selected as examples for further investigation, including Alzheimer's disease (AD, associated_disease_id: C0002395), epilepsy (associated_disease_id: C0014544), major depressive disorder (MDD, associated_disease_id: C1269683), amyotrophic lateral sclerosis (ALS, associated_disease_id: C0002736), and dementia (associated_disease_id: C0497327).

These genes were combined and sorted into different categories. Genes of IS and IS shared genes of AD, epilepsy, MDD, ALS, and dementia were designated as $1,2,3,4,5$, and 6 , respectively.

PPI relationships among disease genes of the abovementioned IS and its associated five CNS diseases were attained from STRING [26]. IS-related DDA network was created with the application of Cytoscape (Version 3.8.0).
2.3. IS-Related Gene Collection and Integration. We manually searched and collected IS-associated genes from public databases, i.e., DisGeNET, Malacards, and OMIM, the different gene symbols were normalized, and duplicated genes were further removed to get three-database-based ISrelated genes. Then, these genes were integrated with genes of index disease (id: C0948008) to get the final genes of IS.

2.4. NBP Regulation on IS-Related DDA Network. NBP potential target genes were integrated into IS DDA network to get the NBP regulation network. The common genes of NBP and diseases may be the potential targets of NBP to show its modulation effects on diseases, i.e., IS, AD, epilepsy, MDD, ALS, and dementia. In order to indicate and visualize the gene-disease association (GDA) among genes and diseases, the DisGeNET Disease Specificity Index (DSI, range 0.25 to 1) and the Disease Pleiotropy Index (DPI, range 0 to 1 ) were cited to evaluate each gene [27]. The common target genes were listed in the bar figure ranked by DSI and DPI. Finally, the NBP-regulated network was constructed with Cytoscape (Version 3.8.0). Target gene nodes were tagged with different colors according to certain gene-disease associations.

2.5. Validation of Potential NBP Targets with Molecular Docking. The potential target genes of NBP with DSI $>0.5$ were selected, which had much bigger specificity to a certain disease. $3 \mathrm{D}$ target protein structures were obtained from the RCSB PDB database, and the target protein structures were pretreated by the application of AutoDock Tools (Version 1.5.6) to generate PDBQT files for molecular docking. NBP .mol2 file was generated with ChemDraw and Chem3D software according to the structure in PubChem (PubChem CID: 61361). The file was further processed and prepared for molecular docking in AutoDock Vina software.

\section{Results}

3.1. NBP Potential Target Identification and Integration. There were a total of 146 potential NBP target genes retrieved and identified from PubMed references. In PharmMapper and DRAR-CPI, 15 potential or putative target genes were manually identified and selected. Another 17 potential target genes were retrieved from the SymMap database. After removing duplicated genes, 175 NBP potential target genes were identified for investigation in deep. The 175 genes were listed in Supplementary Table (Additional file 1).

3.2. IS-Related Gene Collection and Integration. DisGeNET, Malacards, and OMIM were the three sources to obtain IS-associated disease genes. There were 167 IS-related genes attained from DisGeNET, while 168 and 20 genes were obtained from Malacards and OMIM, respectively. These gene names were normalized into official gene symbols, and duplicated symbols were removed. Finally, 312 IS-related genes were obtained for subsequent study (Additional file 2). 


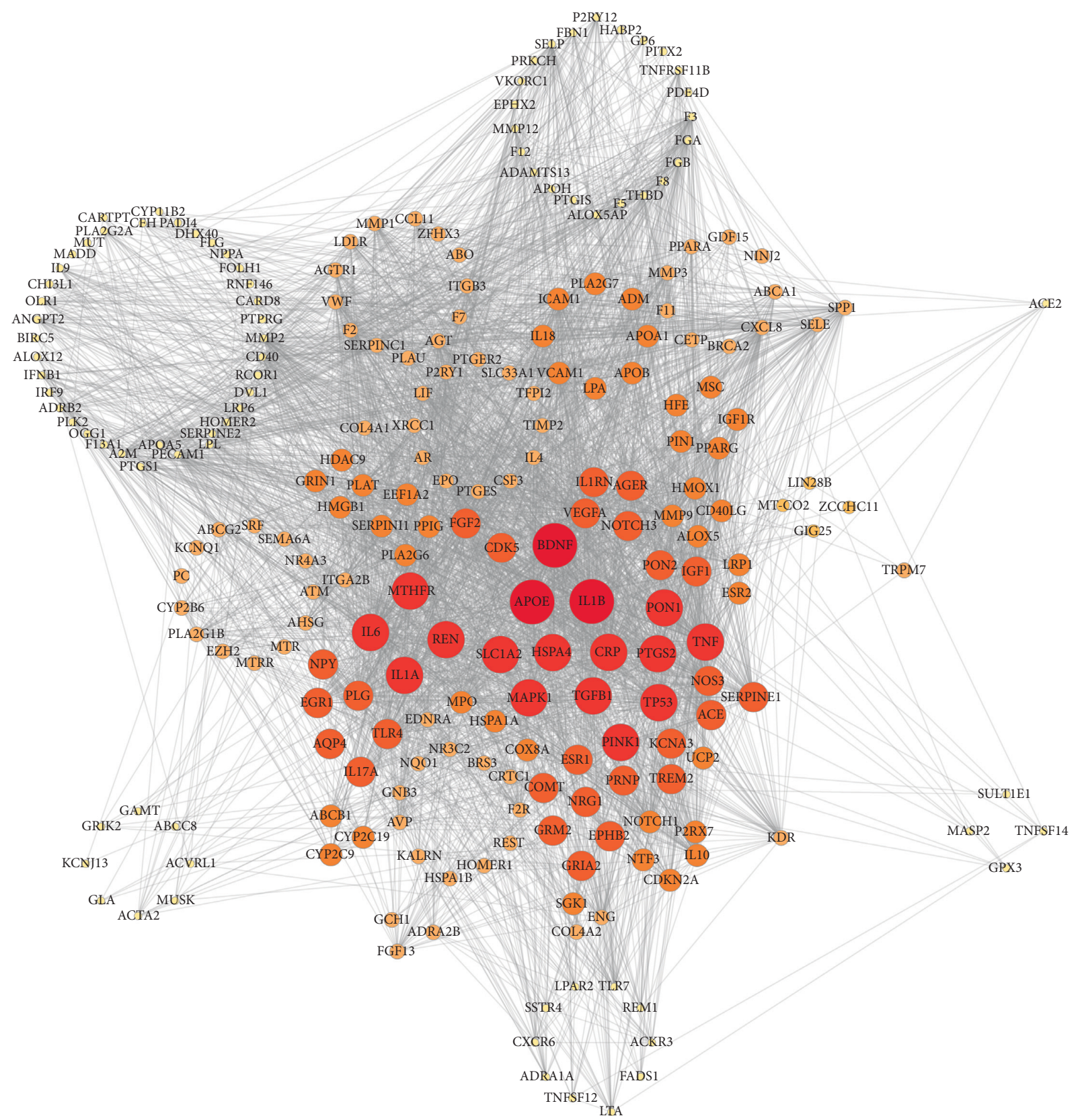

FIgURE 2: Ischemic stroke- (IS-) related disease-disease association (DDA) network. The network contained 241 disease gene nodes and 3433 edges. The node size and color were in proportion to the number of interacted nodes; the more nodes one node linked, the bigger size and deeper color it showed.

3.3. IS-Related DDA Network. "Ischemic stroke" was used as index disease (Index-disease-id: C0948008), and a list of 393 genes was retrieved from DisGeNET ( $5^{\text {th }}$ Dec. 2019) (Additional file 3). These 393 genes were integrated with the abovementioned 312 genes obtained from three sources to get the final 94 IS-associated disease genes (Additional file 4).

Shared gene lists of $\mathrm{AD}$ with IS were retrieved through DDAs (Index-disease-id: C0948008) in DisGeNET, and the same procession was conducted with epilepsy, MDD, ALS, and dementia to obtain their respective shared genes with IS. There were 189 shared genes of $\mathrm{AD}$ and IS, while the numbers for epilepsy, MDD, ALS, and dementia were 77, 75,
67, and 64, respectively. These shared genes were listed in Supplementary Table (Additional file 5). IS DDA network was constructed according to PPI relationships obtained from STRING. The network contained 241 disease gene nodes and 3433 edges. The node size and color were in proportion to the number of interacted nodes; the more nodes one node linked, the bigger size and deeper color it showed (Figure 2).

3.4. NBP Regulation on IS-Related DDA Network. The 175 potential target genes of NBP were integrated into IS DDAs network, and the NBP regulation network was created, in 


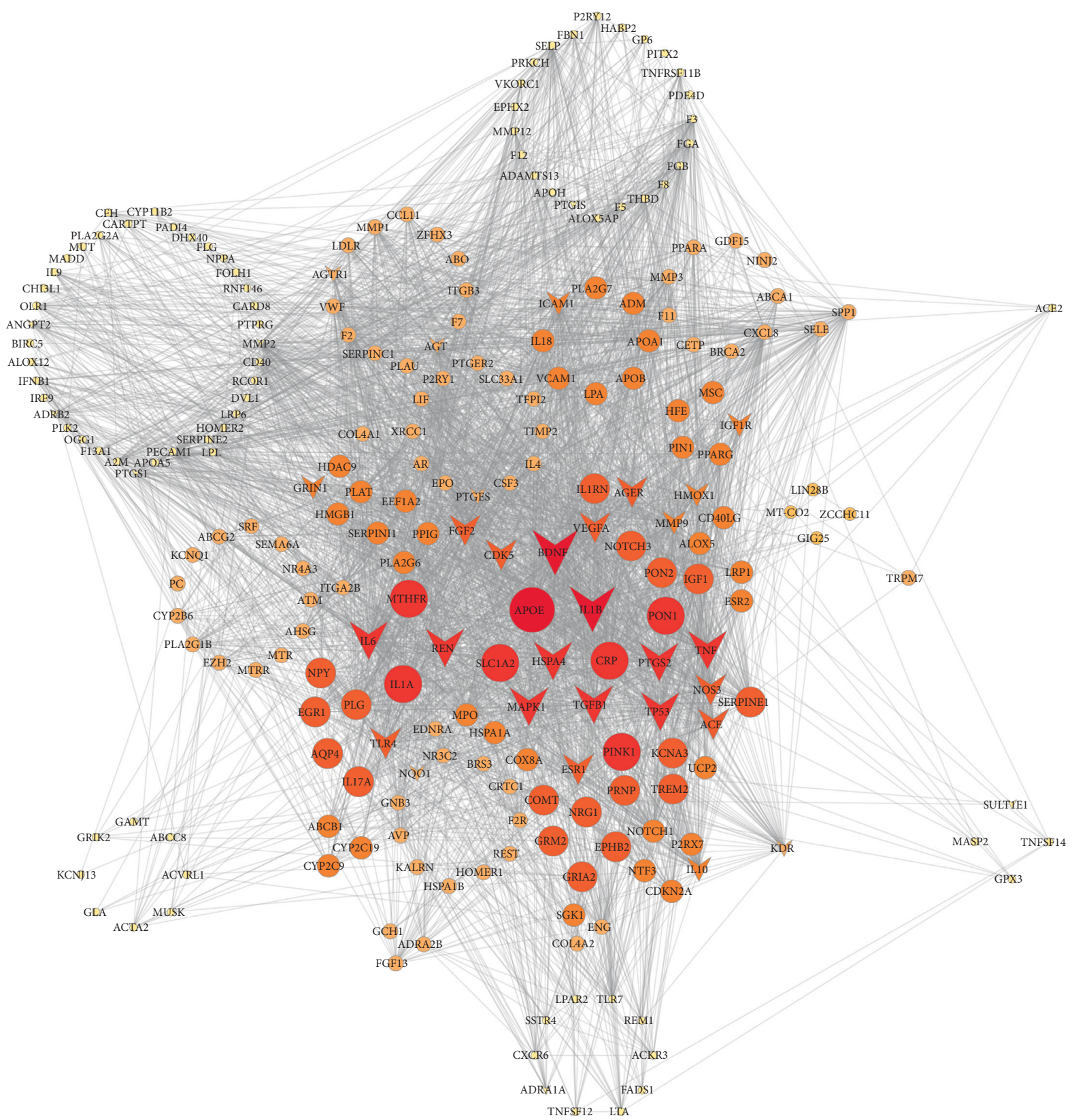

Figure 3: Butylphthalide (NBP) regulation on ischemic stroke- (IS-) related disease-disease association (DDA) network. The 175 potential target genes of NBP were integrated into IS DDA network, and the NBP regulation network was created, in which 36 common genes of NBP and diseases were identified and these 36 nodes were marked as $\mathrm{V}$ shape. The node size and color were in proportion to the number of interacted nodes; the more nodes one node linked, the bigger size and deeper color it showed.

which 36 common genes of NBP and diseases were identified and these 36 nodes were marked as V shape (Figure 3).

As each node may be associated with different diseases, the 36 nodes were marked with different colors according to attributions (Figure 4). In node pie chart color scheme, 1, 2, $3,4,5$, and 6 represent IS, shared genes of AD with IS, shared genes of epilepsy with IS, shared genes of MDD with IS, shared genes of ALS with IS, and shared genes of dementia with IS, respectively.

With network analysis, the associated relationships between the target gene and IS-related disease were predicted; i.e., GRIN1 is associated with AD and epilepsy, PTGES is related to AD and ALS, ADRA1A has a link with depression, CDK5 connects with AD, depression, and ALS, while SULT1E1 may have an association with ALS. These inferred associations could be the underlying basis of repositioning of NBP for IS-associated CNS diseases, and further pieces of evidence were obtained in the discussion section.

The corresponding color was red, blue, green, yellow, cyan, and purple for 1, 2, 3, 4, 5, and 6 (Figure 5(a)). DSI and DPI properties of the 36 node genes were also visualized, in which the 6 NBP potential target genes, i.e., GRIN1, PTGIS, PTGES, ADRA1A, CDK5, and SULT1E1, showed DSI >0.5 (Figure 5(b)). DSI, which is short for disease specificity index 


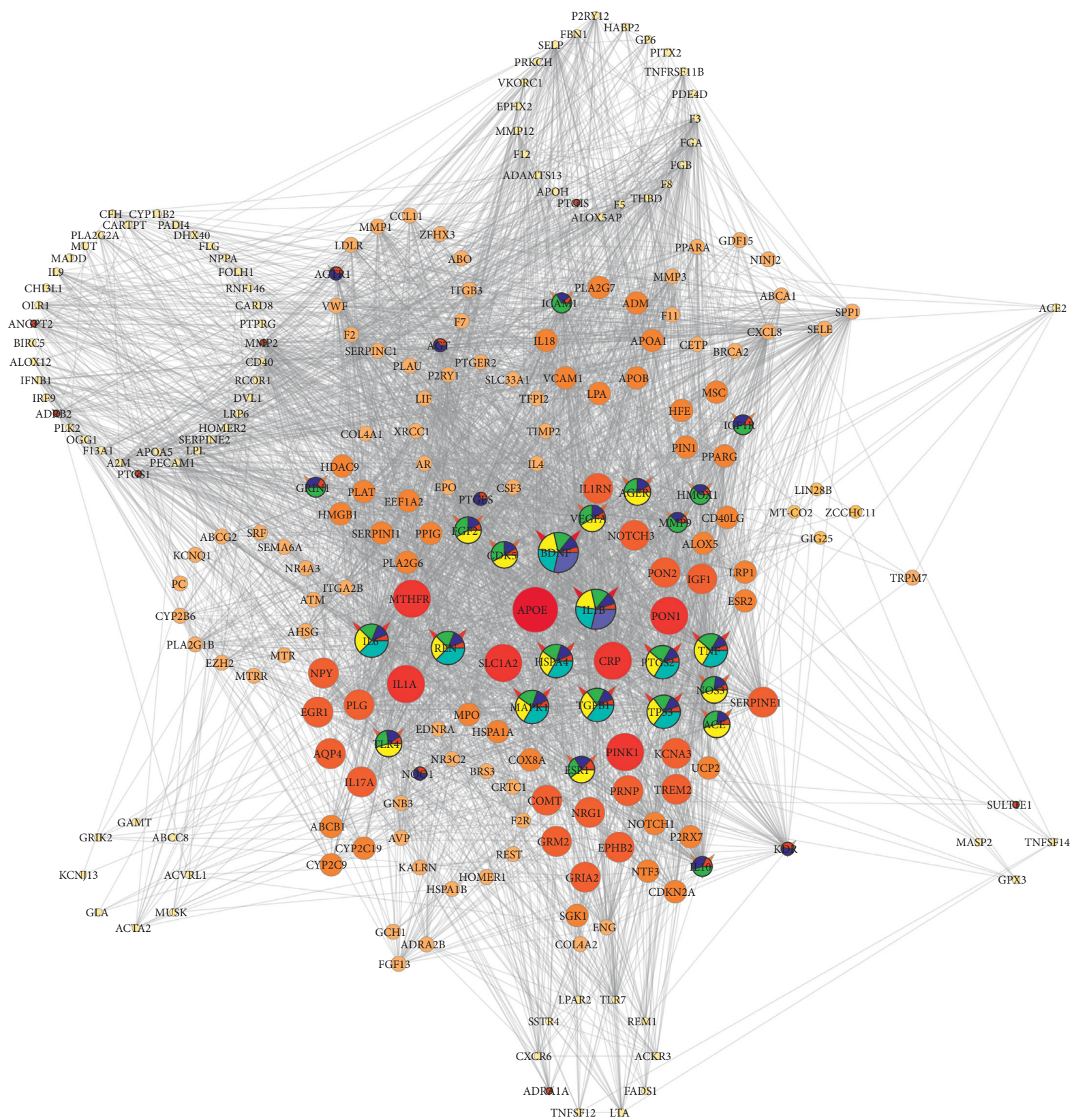

\section{Color scheme:}

$\begin{array}{lllllll}1 & 2 & 3 & 4 & 5 & 6\end{array}$

1. Ischemic stroke, IS

2. Shared genes of AD with IS

4. Shared genes of MDD with IS

5. Shared genes of ALS with IS

6. Shared genes of dementia with Is

FIGURE 4: Pie chart of butylphthalide (NBP) with its regulation on ischemic stroke- (IS-) related disease-disease association (DDA) network. Node pie chart color represents different types of disease genes, i.e., genes of IS (in red), shared genes of AD with IS (in blue), shared genes of epilepsy with IS (in green), shared genes of MDD with IS (in yellow), shared genes of ALS with IS (in cyan), and shared genes of dementia with IS (in purple), respectively. The pie chart was generated in Cytoscape 3.8.0.

in DisGeNET, shows the association degree of a certain gene with several or fewer diseases. And DSI $>0.5$ meant that genes were more related to IS. All of the 6 genes had DPI values of more than 0.4. NBP, as the approved drug by NMPA for IS treatment may show potential regulation effects on several IS-associated CNS diseases through modulation of certain critical DDAs underlying shared genes.
3.5. Validation of Potential NBP Targets with Molecular Docking. Six potential targets of NBP, i.e., GRIN1, PTGIS, PTGES, ADRA1A, CDK5, and SULT1E1, were selected for further validation with in silico molecular docking. Protein structures were obtained from the RCSB PDB database: GRIN1 (PDB: 6IRA, Electron microscopy, $4.50 \AA$ resolution), PTGIS (PDB: 3B6H, X-ray diffraction, $1.62 \AA$ 


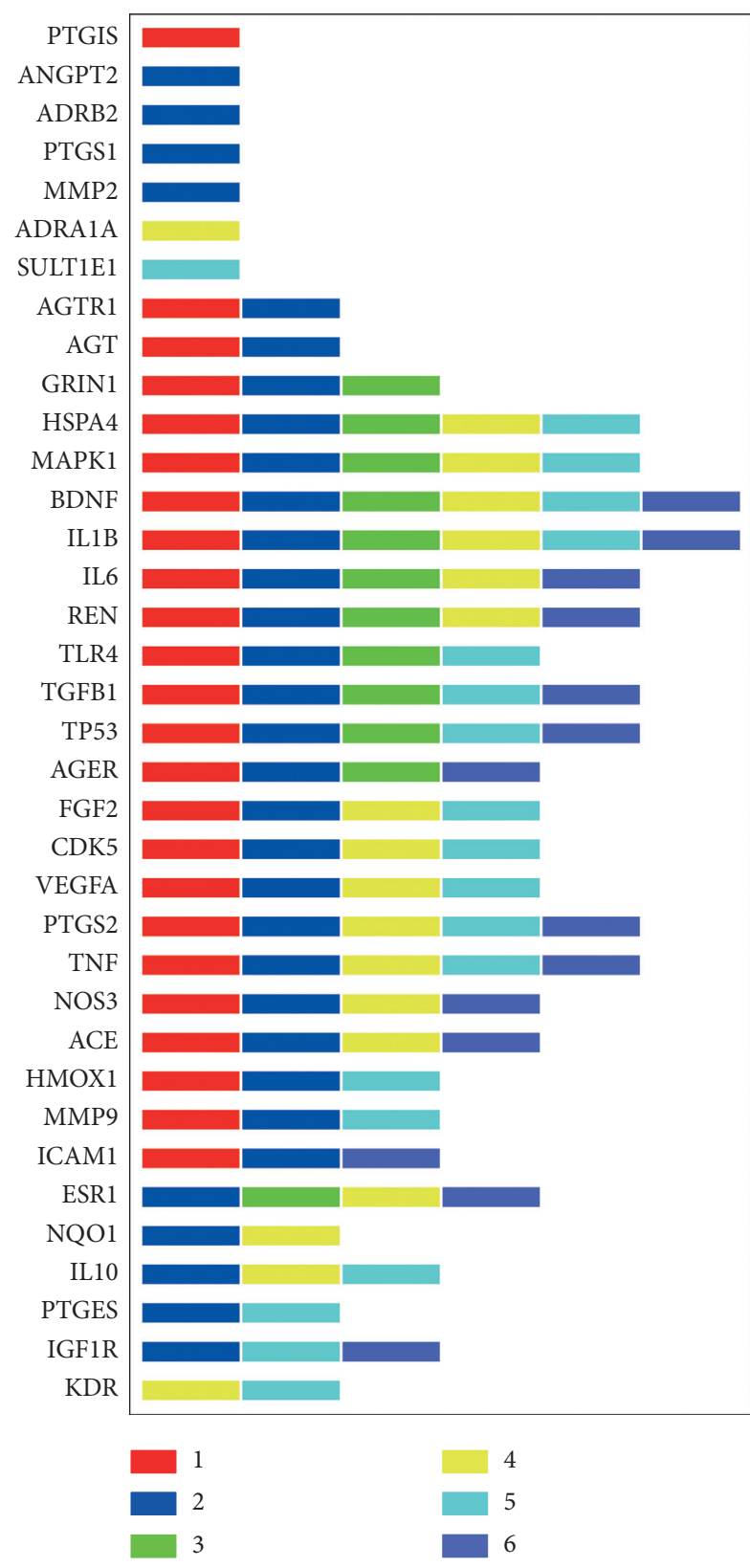

(a)

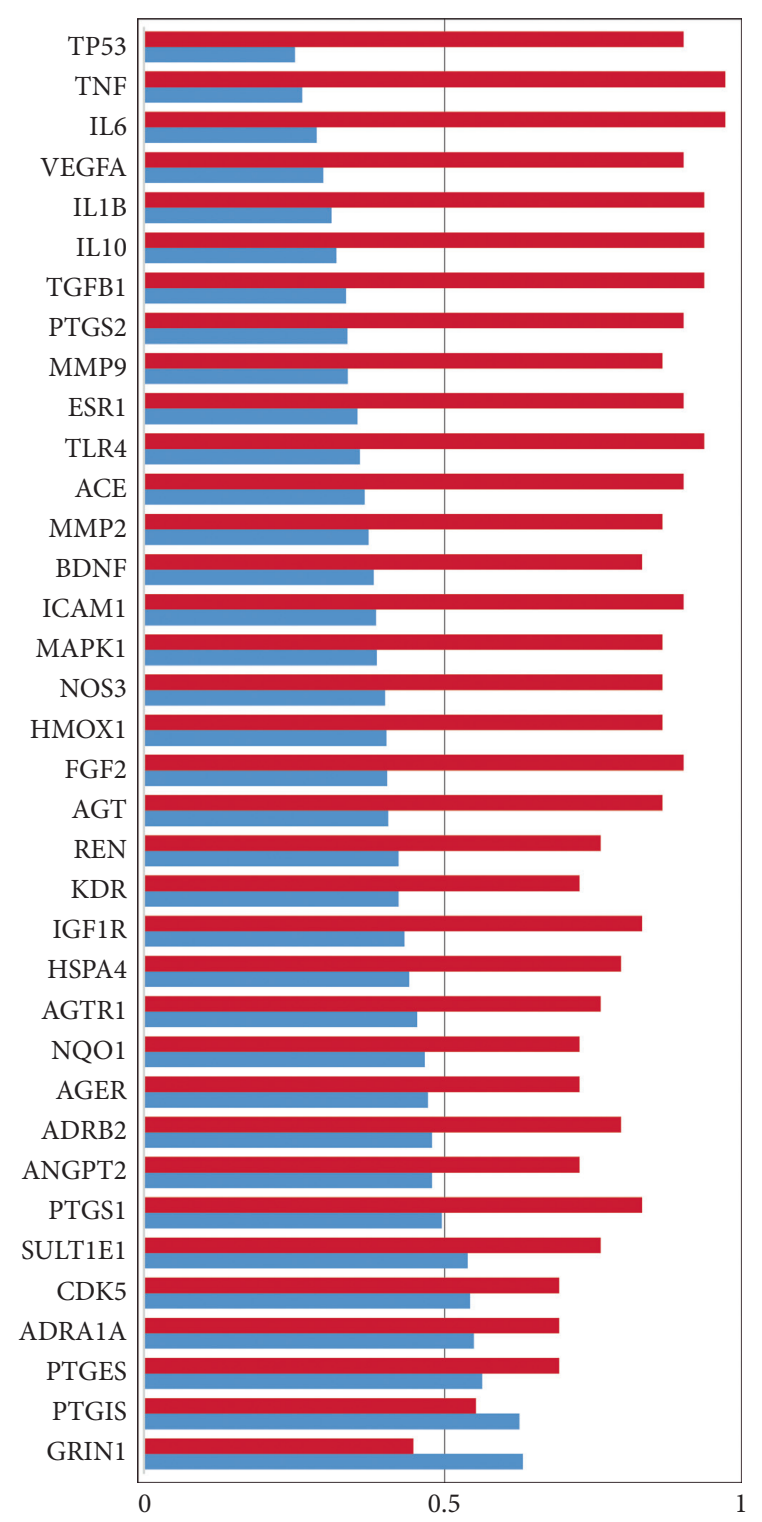

(b)

FIGURE 5: The 36 potential butylphthalide (NBP) target genes in the regulation network of NBP. (a) Gene associations with diseases. 1, 2, 3, 4, 5 , and 6 represent IS, shared genes of AD with IS, shared genes of epilepsy with IS, shared genes of MDD with IS, shared genes of ALS with IS, and shared genes of dementia with IS, respectively. The corresponding color was red, blue, green, yellow, cyan, and purple for 1, 2, 3, 4, 5, and 6. (b) DSI and DPI properties of the 36 potential NBP target genes.

resolution), PTGES (PDB: 3DWW, Electron microscopy, $3.50 \AA$ resolution), CDK5 (PDB: $1 \mathrm{UNL}, \mathrm{X}$-ray diffraction, $2.20 \AA$ resolution), and SULT1E1 (PDB: $1 \mathrm{G} 3 \mathrm{M}, \mathrm{X}$-ray diffraction, $1.70 \AA$ resolution) while the HUMAN-Homology model ADRA1A was achieved from SWISS-MODEL database (P35348, https://swissmodel.expasy.org/repository/ uniprot/P35348?template=4ej4.1.A\&range=22-336).

A docking score was applied to evaluate the binding degree between drug and target. Three levels of the score were used to evaluate the binding activity. When the docking score was less than “ -5 ”, this meant that the drug had a certain binding affinity with the target protein. When the docking score was less than " -7 ", this showed that the drug had good binding activity. When the docking score was less than " -9 ", this indicated that the drug had a strong binding ability [28].

As shown in Figure 6, NBP had a strong binding activity with PTGES (affinity $=-9.2 \mathrm{kcal} / \mathrm{mol}$ ), certain binding ability with GRIN1 (affinity $=-6.7 \mathrm{kcal} / \mathrm{mol}$ ), and good binding ability with PTGIS, ADRA1A, CDK5, and SULT1E1 (affinity $=-7.0,-7.8,-7.5$ and $-7.7 \mathrm{kcal} / \mathrm{mol}$, respectively). 


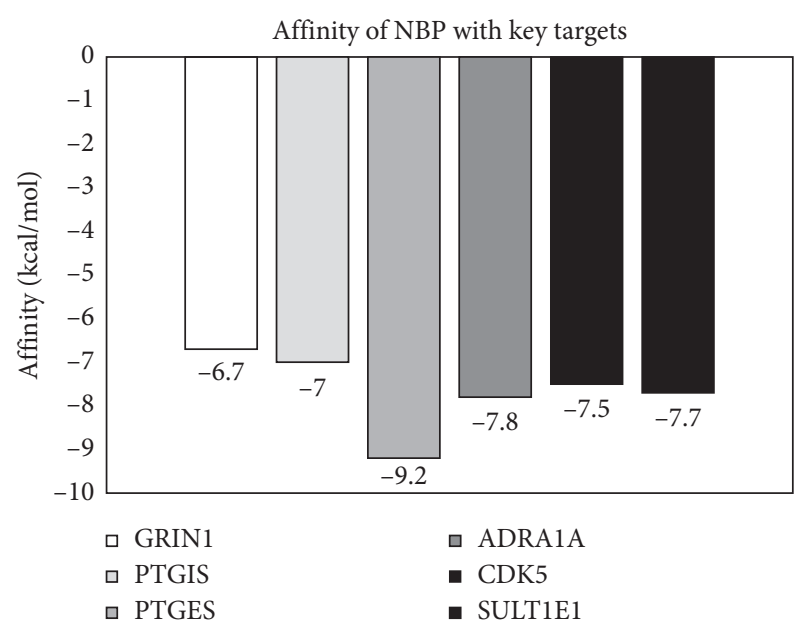

FIgURE 6: Binding affinity of butylphthalide (NBP) with the six key targets. NBP had a strong binding activity with PTGES (affinity $=-9.2 \mathrm{kcal} / \mathrm{mol}$ ), certain binding ability with GRIN1 (affinity $=-6.7 \mathrm{kcal} / \mathrm{mol}$ ), and good binding ability with PTGIS, ADRA1A, CDK5, and SULT1E1 (affinity $=-7.0,-7.8,-7.5$, and $-7.7 \mathrm{kcal} / \mathrm{mol}$, respectively).

The binding modes of NBP with potential target proteins were analyzed by LigPlot+ (Version 2.2). As depicted in Figure 7, GRIN1 had three hydrogen bonds (H-bonds) with NBP (Chain A: Ser 700; Chain B: Asn 432, Lys 457) together with five hydrophobic interactions (Chain A: Arg 673, Glu 698, Thr 701; Chain B: Leu 794, Trp 795). Intriguingly, six conformation structures of NBP possessed a certain binding affinity with GRIN1 or the interface of GRIN1 and GRIN2A in the agonist binding domain $(\mathrm{ABD})$ of a heterotetramer NMDA receptor (details shown in Additional file 6). NBP showed one H-bond with PTGIS (Chain A: Thr 358) and seven hydrophobic interactions (Chain A: Phe 46, Val 74, Tyr 99, Phe 356, Arg 382, Leu 384, and Gly 482). For PTGES, the main interactions between NBP and protein were hydrophobic "bonds" (Chain A: Tyr 80, Leu 83, Phe 84, Phe 87, Val 88 and Phe 91; Chain B: Leu 83, Phe 87 and Phe 91). As for ADRA1A, NBP showed two H-bonds, i.e., Chain A: Ser 188 and Ser 192. Three hydrophobic interactions (Chain A: Asp 106, Val 107, and Cys 110) contributed to the interactions between NBP and ADRA1A. What's more, NBP had one H-bond (Chain A: Cys 83) with CDK5 and 10 hydrophobic interactions (Chain A: Ile 10, Ala 31, Lys 33, Glu 51, Val 64, Phe 80, Phe 82, Leu 133, Ala 143, and Asn 144). And for SULT1E1, NBP possessed two H-bonds and six hydrophobic "bonds" (H-bonds, Chain B: Arg 129; hydrophobic interactions, Chain B: Lys 47, Gly 49, Trp 52, Tyr 192, Phe 228, and Gly 258).

\section{Discussion}

CNS diseases are complex and polygenic diseases lacking clinical effective drugs for treatment. Drug repurposing/ repositioning is one of the feasible and attractive strategies to "recycle" the currently approved drugs. Based on the DDAs, diseases sharing common disease modules, i.e., the underlying disease-associated gene clusters, may have similar symptoms and high comorbidity [3]. IS, as a complex multifactorial disorder to cause brain tissue ischemia and injury, may induce or accompany comorbidities. Human symptoms-disease network [29] showed the IS-related CNS diseases including $\mathrm{AD}$, dementia, epilepsy, glioblastoma/ glioma, motor neuron disease, meningioma, listeriosis, and MDD, with Symptom Similarity Score $\geq 0.2$. Our study set IS-related CNS diseases (i.e., AD, dementia, epilepsy, ALS, and MDD) as examples to elucidate the potential effects of NMPA-approved anti-IS drug NBP on these 5 diseases. NBP showed pleiotropic neuroprotective effects, which may be the underlying molecular mechanisms for its repurposing for the treatment of those five diseases. DisGeNET, Malacards, and OMIM were open-access and high-quality public online disease databases, which contributed to the credibility of the disease-related gene acquisition. STRING was used to retrieve PPI relationships, while network pharmacology methods were applied to construct the DDA network and drug-disease network. With network analysis, critical information contained in networks was extracted, such as the common genes of DDAs, which could be the underlying molecular basis of NBP repositioning.

Through the strategy proposed in this study, six potential targets of NBP, i.e., GRIN1, PTGIS, PTGES, ADRA1A, CDK5, and SULT1E1, were selected for further validation with in silico molecular docking. The pieces of evidence were obtained from references to support these associations among targets and diseases as follows.

4.1. GRIN1. GRIN1 is an indispensable component of the heterotetramer of NMDA receptor complexes and plays a critical role in the plasticity of synapses. Our results indicated that it had associations with IS, AD, and epilepsy. The learning and memory-related GRIN1 gene was found downregulated in ovariectomized $\mathrm{AD}$ rats [30]. Studies reported that GRIN1 was one of the top 25 core genes of the AD network [31]. Another study showed GRIN1 as one of the target genes of the approved anti-AD drug memantine [32].

GRIN1 was also one of the top candidate genes for epileptogenesis [33]. The whole-exome sequencing analysis of patients found that GRIN1 mutations caused seizures and movement disorders [34]. GRIN1 mutation associated with intellectual disability alters NMDA receptor trafficking and function [35]. $70 \%-80 \%$ of epilepsy is attributed to genetic factors, the GRIN1 gene encodes the NMDA receptor GluN1 subunit, and the NMDA receptor has an important role in epilepsy [36].

4.2. PTGES. Molecular docking results indicated that NBP had a strong binding activity with PTGES (affinity $=-9.2 \mathrm{kcal} / \mathrm{mol}$ ). As we know, PTGES encodes prostaglandin E synthase, which catalyzes the glutathionedependent oxidoreduction of prostaglandin endoperoxide $\mathrm{H}_{2}\left(\mathrm{PGH}_{2}\right)$ to prostaglandin $\mathrm{E}_{2}\left(\mathrm{PGE}_{2}\right)$ in response to inflammatory stimuli. Studies showed that microsomal prostaglandin E synthase 1 (mPGES-1) expression was significantly elevated in middle frontal gyrus tissues of $\mathrm{AD}$ 


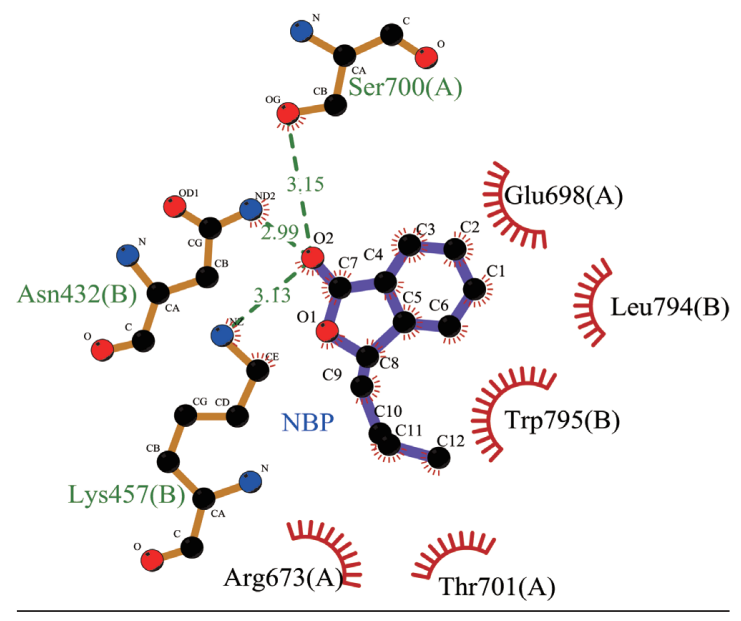

(a)

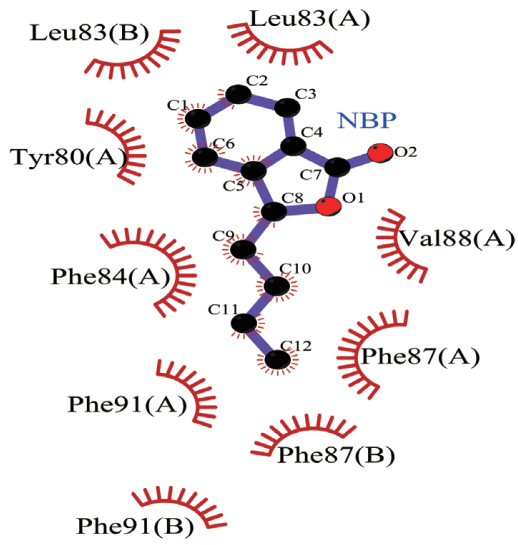

(c)

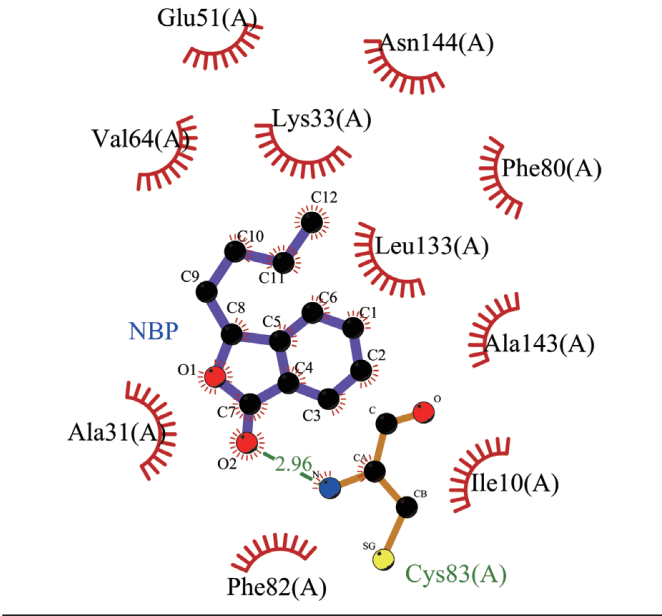

(e)

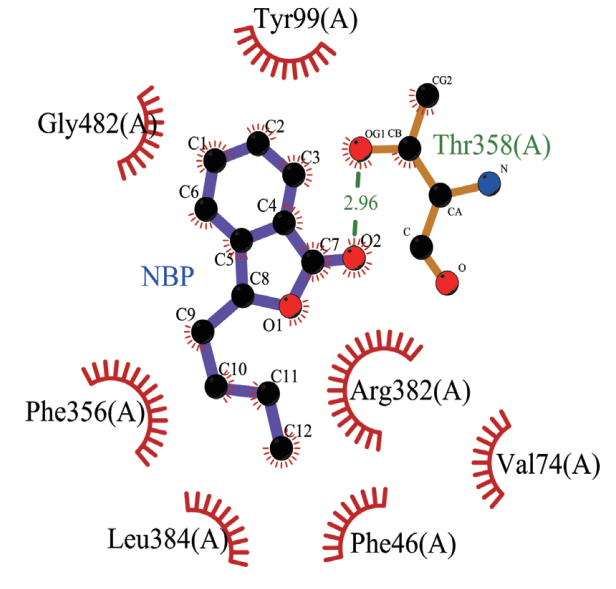

(b)

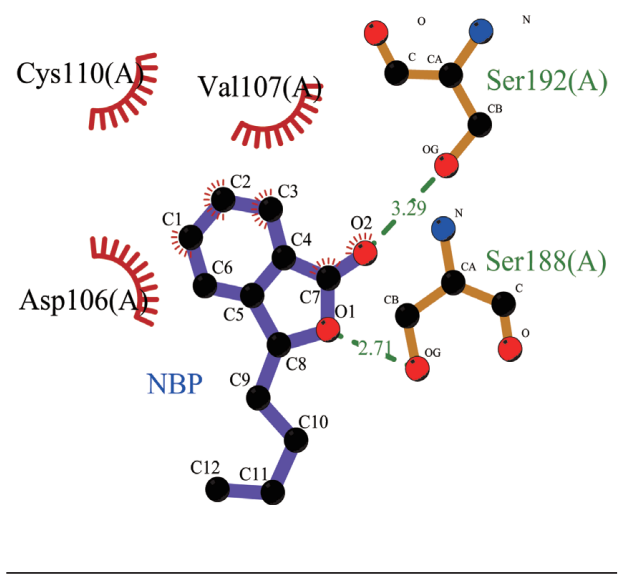

(d)

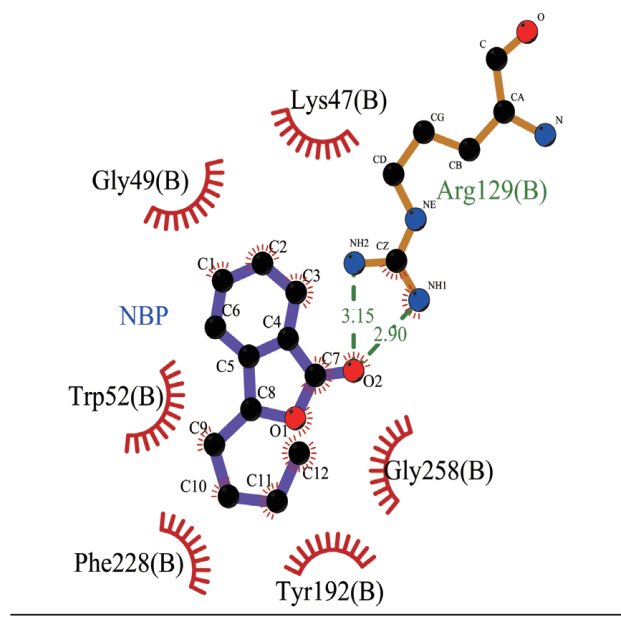

(f)

Figure 7: The binding modes of NBP with potential target proteins. Binding modes were analyzed by LigPlot+ (Version 2.2). The green dashed lines stand for hydrogen bonds (H-bonds), while the green numbers on lines stand for distances $(\AA)$. The purple bonds stand for ligand bonds, while the brown color stands for nonligand bonds. And the brick red color represents hydrophobic "bonds." For atoms, the blue colors stand for nitrogen atoms, the red color stands for oxygen atoms, the yellow color was for sulfur atoms, and the black color represents carbon atoms. (a) NBP_GRIN1. (b) NBP_PTGIS. (c) NBP_PTGES. (d) NBP_ADRA1A. (e) NBP_CDK5. (f) NBP_SULT1E1. 
patients compared with controls, and the proinflammatory $\mathrm{PGE}_{2}$ [37] was found to be elevated in the cerebrospinal fluid early in $\mathrm{AD}$ [38]. However, the effect of $\mathrm{PGE}_{2}$ depends on its concentration and to which receptor it binds [39]. Reports indicated that mPGES- 1 was induced in $\mathrm{A} \beta$-mediated neuronal cell death, and $\mathrm{PGE}_{2}$ was an important factor involved in A $\beta$-induced neurotoxicity [40]. mPGES-1 may play a role in $\mathrm{AD}$ pathology [41].

ALS is a progressive neurodegenerative disorder characterized by the selective death of motor neurons. $\mathrm{PGE}_{2}$ was elevated in the serum and CSF of ALS patients, and mPGES1 inhibition may reduce microglial activation and motor neuron loss [42]. Thus, mPGES-1 in motor neurons may play a role in the pathogenesis of ALS [43]. Another work reported that the concurrent inhibition of mPGES-1 and free radicals would be a promising strategy to combat neurodegeneration in ALS [44].

4.3. ADRA1A. As for depression disorder, tricyclic antidepressant (TCA) drugs can also target $\alpha_{1}$-adrenergic receptor (AR) as antagonists, which may be the underlying mechanisms for therapy [45]. Further investigations into the subtypes of $\alpha_{1}$-AR indicated that $\alpha_{1 \mathrm{~A}}$-AR may be a useful therapeutic target for the treatment of depression [46]. What's more, norepinephrine (NE) plays an important role in behavior and cognition and involves depression regulation [47]. While $\alpha_{1 \mathrm{~A}} \mathrm{AR}$ was associated with antidepressant-like effects, chronic $\alpha_{1 \mathrm{~B}}-\mathrm{AR}$ stimulation showed prodepressant [48]. Deeper investigations deserve to be designed and conducted to clarify the involvement role of $\alpha_{1 \mathrm{~A}}-\mathrm{AR}$ against depression.

4.4. CDK5. CKD5 is a versatile kinase showing a pivotal role in modulating the function of postmitotic neurons in the developing CNS; the dysregulation of CDK5 is involved in the pathology of neurodegenerative diseases, such as AD, ALS, and IS $[49,50]$. CDK5 deregulation contributes to the pathogenesis of $\mathrm{AD}$ through inducing deposition of $\mathrm{A} \beta$ in senile plaques and intracellular accumulations of hyperphosphorylated tau. The balance of CDK5/P35 and CDK5/ P25 is crucial for neuronal migration and differentiation, neurite outgrowth, synaptic growth, and functions. Elevated and sustained Cdk5/p25 activity consequently leads to DNA damage, cell death, and neurodegeneration, whereas the $\mathrm{Cdk} 5 / \mathrm{p} 35$ complex is neuroprotective $[51,52]$. Thus, CDK5 could be a potential therapeutic target for AD [53-55].

As suppression of overactivated or deregulated CDK5 offers neuroprotection and prevention of motor neuron loss. ALS, the progressive and fatal neurodegenerative disorder leading to muscle atrophy, loss of movement, and eventually death, could gain benefits through inhibition of CDK5 [51, 56-58].

CDK5 was reported to have an association with depression, but the underlying mechanisms may be complex. CDK5 in the dentate gyrus was found to participate in the process of depressive-like behavior in rats. Inhibition of CDK5 in the dentate gyrus contributed to the antidepressant actions and ameliorated the depressive-like symptoms [59].
CDK5 loss of function in the ventral tegmental area (VTA) induced anxiety- and depressive-like behaviors in mouse models which were associated with inhibited tyrosine hydroxylase phosphorylation at Ser31 and Ser 40 [60]. In an MDD mouse model, studies indicated that CDK5-mediated phosphorylation of Sirt 2 at Ser 368 and 372 in amygdala accounting for social defeat stress induced depressive-like behavior. Thus, inhibition of CDK5-dependent Sirt2 phosphorylation at Ser 368 and 372 may be a promising strategy for antidepression therapy development [61]. Another study showed Cdk5 in the nucleus accumbens as a critical contributor to depressive-like behaviors in Huntington's disease mouse models [62].

4.5. SULT1E1. Estrogen sulfotransferase (EST, encoded by SULT1E1) catalyzes the sulfoconjugation and inactivation of estrogen. Reports showed that estrogen exerts neuroprotective and anti-inflammatory effects in ALS $[63,64]$. As EST mediates the metabolic deactivation of estrogen [65], drugs targeting EST may have potential regulation effects against ALS.

In summary, our study proposed a network pharmacology and molecular docking integrated strategy to predict several critical targets of NBP with drug repurposing potential; in silico molecular docking and literature validations were conducted to support these findings. However, more works in deep are warranted to design and investigate the associations between NBP and AD, epilepsy, ALS, and depression.

\section{Conclusions}

NBP, an NMPA-approved drug for IS treatment, shows multitarget and pleiotropic effects. IS has DDAs with several CNS diseases, including AD, ALS, epilepsy, MDD, and dementia. Network pharmacology could facilitate the construction of NBP-regulated disease networks to explore the underlying molecular mechanisms of potential effects. 36 potential target genes of NBP were identified for ISassociated CNS diseases, and 6 of them, i.e., GRIN1, PTGIS, PTGES, ADRA1A, CDK5, and SULT1E1 were validated by the application of in silico molecular docking. All of the six targets indicated certain to good binding affinity with NBP. References were retrieved to further validate the association between target genes and IS-related CNS diseases. Based on network analysis and validations, NBP may have the potential to be repositioned for AD, epilepsy, ALS, and depression treatments.

In summary, our study has proposed a drug-targetdisease integrating method to predict the drug repurposing potentials to other associated diseases by the application of network pharmacology and molecular docking, which could be an attractive alternative to facilitate the development of CNS disease therapies. As this drug repositioning strategy is based on databases, in silico molecular docking, and published references, further in-deep investigations are warranted to be carefully designed and conducted. 


\section{Data Availability}

All data are available within the article and are shown in figures and supplemental files.

\section{Conflicts of Interest}

All authors declare no conflicts of interest.

\section{Authors' Contributions}

QQZ and XL conceived and designed the study. QQZ, BZ, and XL performed the experiments. QQZ, PPF, and XL performed the data analysis. QQZ and XL prepared the figures and tables. BZ and PPF corrected the manuscript. All authors are responsible for reviewing data. All authors read and approved the final manuscript.

\section{Acknowledgments}

The authors thank Prof. Xiaohui Fan (the Deputy Dean of the School of Pharmaceutical Sciences, Zhejiang University; the Deputy Director of the Institute of Pharmaceutical Informatics, and the Deputy Director of Tianjin Modern Chinese Medicine Collaborative Innovation Center; E-mail: fanxh@zju.edu.cn) for his kind and helpful suggestions in study design and linguistic assistance during the investigation and preparation of this manuscript. This work was supported by the Natural Science Foundation of Zhejiang Province (LYY19H280005 and LQ17H280001), the Scientific Foundation in TCM of Zhejiang Province (2017ZQ008), the Natural Science Foundation of Hangzhou Medical College (2015B01), the Zhejiang Pharmaceutical Association (2018ZYY11), and the "13th Five-Year" Chinese Medicine Key Discipline in Zhejiang ProvinceChinese Medicine Quality and Functional Evaluation (2017XK-A43 to J. Shi).

\section{Supplementary Materials}

Additional file 1: the 175 NBP genes. Additional file 2: 312 IS-related genes obtained from databases of Malacards, DisGeNET, and OMIM. Additional file 3: genes of ischemic stroke collected from DisGeNET database (Index_disease_id: C0948008). Additional file 4: final 94 ischemic stroke genes after gene symbols are normalized and duplicated genes are removed. Additional file 5: shared genes of $\mathrm{AD}$, epilepsy, MDD, ALS, and dementia with IS, respectively. Additional file 6: six conformations of GRIN1 in molecular docking with NBP. (Supplementary Materials)

\section{References}

[1] F. Gasparini and T. Di Paolo, "Drug repurposing: old drugs, new tricks to fast track drug development for the brain," Neuropharmacology, vol. 147, pp. 1-3, 2019.

[2] H. Mei, T. Xia, G. Feng, J. Zhu, S. M. Lin, and Y. Qiu, "Opportunities in systems biology to discover mechanisms and repurpose drugs for CNS diseases," Drug Discovery Today, vol. 17, no. 21-22, pp. 1208-1216, 2012.
[3] J. Menche, A. Sharma, M. Kitsak et al., "Disease networks. Uncovering disease-disease relationships through the incomplete interactome," Science, vol. 347, no. 6224, Article ID 1257601, 2015.

[4] C. G. Parsons, "CNS repurposing-potential new uses for old drugs: examples of screens for Alzheimer's disease, Parkinson's disease and spasticity," Neuropharmacology, vol. 147, pp. 4-10, 2019.

[5] N. Nosengo, "Can you teach old drugs new tricks?," Nature, vol. 534, no. 7607, pp. 314-316, 2016.

[6] D. C. Hess and S. C. Fagan, "Repurposing an old drug to improve the use and safety of tissue plasminogen activator for acute ischemic stroke: minocycline," Pharmacotherapy, vol. 30, no. 7, pp. 55S-61S, 2010.

[7] A. Corbett, J. Pickett, A. Burns et al., "Drug repositioning for Alzheimer's disease," Nature Reviews Drug Discovery, vol. 11, no. 11, pp. 833-846, 2012.

[8] S. Kumar, S. Chowdhury, and S. Kumar, "In silico repurposing of antipsychotic drugs for Alzheimer's disease," BMC Neuroscience, vol. 18, no. 1, p. 76, 2017.

[9] M. Hassan, H. Raza, M. A. Abbasi, A. A. Moustafa, and S.-Y. Seo, "The exploration of novel Alzheimer's therapeutic agents from the pool of FDA approved medicines using drug repositioning, enzyme inhibition and kinetic mechanism approaches," Biomedicine \& Pharmacotherapy, vol. 109, pp. 2513-2526, 2019.

[10] L. Brueggeman, M. L. Sturgeon, R. M. Martin et al., "Drug repositioning in epilepsy reveals novel antiseizure candidates," Annals of Clinical and Translational Neurology, vol. 6, no. 2, pp. 295-309, 2019.

[11] C. Moran, M. L. Callisaya, V. Srikanth, and Z. Arvanitakis, "Diabetes therapies for dementia," Current Neurology and Neuroscience Reports, vol. 19, no. 8, p. 58, 2019.

[12] H. Yoshino, "Edaravone for the treatment of amyotrophic lateral sclerosis," Expert Review of Neurotherapeutics, vol. 19, no. 3, pp. 185-193, 2019.

[13] Q. q. Zhao, X. Li, L. p. Luo, Y. Qian, Y. l. Liu, and H. t. Wu, "Repurposing of approved cardiovascular drugs against ischemic cerebrovascular disease by disease-disease associated network-assisted prediction," Chemical and Pharmaceutical Bulletin, vol. 67, no. 1, pp. 32-40, 2019.

[14] A. L. Hopkins, "Network pharmacology: the next paradigm in drug discovery," Nature Chemical Biology, vol. 4, no. 11, pp. 682-690, 2008.

[15] A. Anighoro, J. Bajorath, and G. Rastelli, "Polypharmacology: challenges and opportunities in drug discovery," Journal of Medicinal Chemistry, vol. 57, no. 19, pp. 7874-7887, 2014.

[16] P. Zhang, R. Xu, Y. Guo et al., "DL-3-n-butylphthalide promotes dendrite development in cortical neurons subjected to oxygen-glucose deprivation/reperfusion," Cell Biology International, vol. 42, no. 8, pp. 1041-1049, 2018.

[17] Y. Chen, T. Wu, H. Li et al., "Dl-3-n-butylphthalide exerts dopaminergic neuroprotection through inhibition of neuroinflammation," Frontiers in Aging Neuroscience, vol. 11, p. 44, 2019.

[18] X. L. Niu, X. Jiang, G. D. Xu et al., "DL-3-n-butylphthalide alleviates vascular cognitive impairment by regulating endoplasmic reticulum stress and the Shh/Ptch1 signalingpathway in rats," Journal of Cellular Physiology, vol. 234, no. 8, pp. 12604-12614, 2019.

[19] X. Wang, Y. Shen, S. Wang et al., "PharmMapper 2017 update: a web server for potential drug target identification with a comprehensive target pharmacophore database," Nucleic Acids Research, vol. 45, no. W1, pp. W356-W360, 2017. 
[20] H. Luo, J. Chen, L. Shi et al., "DRAR-CPI: a server for identifying drug repositioning potential and adverse drug reactions via the chemical-protein interactome," Nucleic Acids Research, vol. 39, no. 2, pp. W492-W498, 2011.

[21] Y. Wu, F. Zhang, K. Yang et al., "SymMap: an integrative database of traditional Chinese medicine enhanced by symptom mapping," Nucleic Acids Research, vol. 47, no. D1, pp. D1110-D1117, 2019.

[22] N. Rappaport, M. Twik, I. Plaschkes et al., "MalaCards: an amalgamated human disease compendium with diverse clinical and genetic annotation and structured search," Nucleic Acids Research, vol. 45, no. D1, pp. D877-D887, 2017.

[23] A. Hamosh, A. F. Scott, J. S. Amberger, C. A. Bocchini, and V. A. McKusick, "Online Mendelian Inheritance in Man (OMIM), a knowledgebase of human genes and genetic disorders," Nucleic Acids Research, vol. 33, pp. D514-D517, 2005.

[24] O. Trott and A. J. Olson, "AutoDock Vina: improving the speed and accuracy of docking with a new scoring function, efficient optimization, and multithreading," Journal of Computational Chemistry, vol. 31, no. 2, pp. 455-461, 2010.

[25] P. Shannon, A. Markiel, O. Ozier et al., "Cytoscape: a software environment for integrated models of biomolecular interaction networks," Genome Research, vol. 13, no. 11, pp. 2498-2504, 2003.

[26] D. Szklarczyk, A. L. Gable, D. Lyon et al., "STRING v11: protein-protein association networks with increased coverage, supporting functional discovery in genome-wide experimental datasets," Nucleic Acids Research, vol. 47, no. D1, pp. D607-D613, 2019.

[27] J. Piñero, J. M. Ramírez-Anguita, J. Saüch-Pitarch et al., "The DisGeNET knowledge platform for disease genomics: 2019 update," Nucleic Acids Research, vol. 48, no. D1, pp. D845-D55, 2020.

[28] T. Gaillard, "Evaluation of AutoDock and AutoDock Vina on the CASF-2013 benchmark," Journal of Chemical Information and Modeling, vol. 58, no. 8, pp. 1697-1706, 2018.

[29] X. Zhou, J. Menche, A. L. Barabasi, and A. Sharma, "Human symptoms-disease network," Nature Communications, vol. 5, Article ID 4212, 2014.

[30] C. Agca, D. Klakotskaia, E. G. Stopa, T. R. Schachtman, and Y. Agca, "Ovariectomy influences cognition and markers of Alzheimer's disease," Journal of Alzheimer's Disease, vol. 73, no. 2, pp. 529-541, 2020.

[31] P. Hallock and M. A. Thomas, "Integrating the Alzheimer's disease proteome and transcriptome: a comprehensive network model of a complex disease," OMICS, vol. 16, no. 1-2, pp. 37-49, 2012.

[32] M. K. Kwok, S. L. Lin, and C. M. Schooling, "Re-thinking Alzheimer's disease therapeutic targets using gene-based tests," EBioMedicine, vol. 37, pp. 461-470, 2018.

[33] D. A. Forero, "Functional genomics of epileptogenesis in animal models and humans," Cellular and Molecular Neurobiology, 2020.

[34] C. Ohba, M. Shiina, J. Tohyama et al., "GRIN1 mutations cause encephalopathy with infantile-onset epilepsy, and hyperkinetic and stereotyped movement disorders," Epilepsia, vol. 56, no. 6, pp. 841-848, 2015.

[35] W. Chen, C. Shieh, S. A. Swanger et al., "GRIN1 mutation associated with intellectual disability alters NMDA receptor trafficking and function," Journal of Human Genetics, vol. 62, no. 6, pp. 589-597, 2017.

[36] X. X. Xu and J. H. Luo, "Mutations of N-methyl-D-aspartate receptor subunits in epilepsy," Neuroscience Bulletin, vol. 34, no. 3, pp. 549-565, 2018.
[37] Y. Ikeda-Matsuo, "The role of mPGES-1 in inflammatory brain diseases," Biological \& Pharmaceutical Bulletin, vol. 40, no. 5, pp. 557-563, 2017.

[38] U. A. Chaudhry, H. Zhuang, B. J. Crain, and S. Doré, "Elevated microsomal prostaglandin-E synthase-1 in Alzheimer's disease," Alzheimer's \& Dementia, vol. 4, no. 1, pp. 6-13, 2008.

[39] U. Chaudhry, H. Zhuang, and S. Doré, "Microsomal prostaglandin E synthase-2: cellular distribution and expression in Alzheimer's disease," Experimental Neurology, vol. 223, no. 2, pp. 359-365, 2010.

[40] Y. Kuroki, Y. Sasaki, D. Kamei et al., "Deletion of microsomal prostaglandin E synthase-1 protects neuronal cells from cytotoxic effects of $\beta$-amyloid peptide fragment 31-35," Biochemical and Biophysical Research Communications, vol. 424, no. 3, pp. 409-413, 2012.

[41] Y. Akitake, Y. Nakatani, D. Kamei et al., "Microsomal prostaglandin E synthase-1 is induced in Alzheimer's disease and its deletion mitigates Alzheimer's disease-like pathology in a mouse model," Journal of Neuroscience Research, vol. 91, no. 7, pp. 909-919, 2013.

[42] I. V. Lima, L. F. Bastos, M. Limborco-Filho, B. L. Fiebich, and A. C. de Oliveira, "Role of prostaglandins in neuroinflammatory and neurodegenerative diseases," Mediators of Inflammation, vol. 2012, Article ID 946813, 13 pages, 2012.

[43] H. Miyagishi, Y. Kosuge, K. Ishige, and Y. Ito, "Expression of microsomal prostaglandin E synthase- 1 in the spinal cord in a transgenic mouse model of amyotrophic lateral sclerosis," Journal of Pharmacological Sciences, vol. 118, no. 2, pp. 225236, 2012.

[44] J. H. Shin, Y. A. Lee, J. K. Lee et al., "Concurrent blockade of free radical and microsomal prostaglandin E synthase-1mediated PGE2 production improves safety and efficacy in a mouse model of amyotrophic lateral sclerosis," Journal of Neurochemistry, vol. 122, no. 5, pp. 952-961, 2012.

[45] Z.-j. Chen and K. P. Minneman, "Recent progress in alpha1adrenergic receptor research," Acta Pharmacologica Sinica, vol. 26, no. 11, pp. 1281-1287, 2005.

[46] V. A. Doze, E. M. Handel, K. A. Jensen et al., " $\alpha 1 \mathrm{~A}-$ and $\alpha 1 \mathrm{~B}-$ adrenergic receptors differentially modulate antidepressantlike behavior in the mouse," Brain Research, vol. 1285, pp. 148-157, 2009.

[47] O. Borodovitsyna, M. Flamini, and D. Chandler, "Noradrenergic modulation of cognition in health and disease," Neural Plasticity, vol. 2017, Article ID 6031478, 14 pages, 2017.

[48] D. M. Perez and V. A. Doze, "Cardiac and neuroprotection regulated by $\alpha 1$-adrenergic receptor subtypes," Journal of Receptors and Signal Transduction, vol. 31, no. 2, pp. 98-110, 2011.

[49] R. Dhavan and L. H. Tsai, “A decade of CDK5,” Nature Reviews Molecular Cell Biology, vol. 2, no. 10, pp. 749-759, 2001.

[50] J. P. Lopes and P. Agostinho, "Cdk5: multitasking between physiological and pathological conditions," Progress in Neurobiology, vol. 94, no. 1, pp. 49-63, 2011.

[51] S. C. Su and L. H. Tsai, "Cyclin-dependent kinases in brain development and disease," Annual Review of Cell and Developmental Biology, vol. 27, no. 1, pp. 465-491, 2011.

[52] S. L. Liu, C. Wang, T. Jiang, L. Tan, A. Xing, and J. T. Yu, “The role of Cdk5 in Alzheimer's disease," Molecular Neurobiology, vol. 53, no. 7, pp. 4328-4342, 2016.

[53] A. B. Allnutt, A. K. Waters, S. Kesari, and V. M. Yenugonda, "Physiological and pathological roles of Cdk5: potential directions for therapeutic targeting in neurodegenerative disease," ACS Chemical Neuroscience, vol. 11, no. 9, pp. 1218-1230, 2020. 
[54] Y. Qi, L. Guo, Y. Jiang, Y. Shi, H. Sui, and L. Zhao, "Brain delivery of quercetin-loaded exosomes improved cognitive function in $\mathrm{AD}$ mice by inhibiting phosphorylated tau-mediated neurofibrillary tangles," Drug Delivery, vol. 27, no. 1, pp. 745-755, 2020.

[55] Y. Wang, J. Zhao, F. L. Guo et al., "Metformin ameliorates synaptic defects in a mouse model of $\mathrm{AD}$ by inhibiting Cdk 5 activity," Frontiers in Cellular Neuroscience, vol. 14, p. 170, 2020.

[56] G. Mushtaq, N. H. Greig, F. Anwar et al., "Neuroprotective mechanisms mediated by CDK5 inhibition," Current Pharmaceutical Design, vol. 22, no. 5, pp. 527-534, 2016.

[57] H. Patzke and L. H. Tsai, "Cdk5 sinks into ALS," Trends in Neurosciences, vol. 25, no. 1, pp. 8-10, 2002.

[58] B. K. Skuntz, M. Prochazkova, S. Kesavapany et al., "Overexpression of the Cdk5 inhibitory peptide in motor neurons rescue of amyotrophic lateral sclerosis phenotype in a mouse model," Human Molecular Genetics, vol. 28, no. 19, pp. 3175-3187, 2019.

[59] W. L. Zhu, H. S. Shi, S. J. Wang et al., "Increased Cdk5/p35 activity in the dentate gyrus mediates depressive-like behaviour in rats," The International Journal of Neuropsychopharmacology, vol. 15, no. 6, pp. 795-809, 2012.

[60] P. Zhong, X. Liu, Z. Zhang et al., "Cyclin-dependent kinase 5 in the ventral tegmental area regulates depression-related behaviors," Journal of Neuroscience, vol. 34, no. 18, pp. 6352-6366, 2014.

[61] Z. Zhang, P. Zhang, G. J. Qi et al., "CDK5-mediated phosphorylation of Sirt2 contributes to depressive-like behavior induced by social defeat stress," Biochimica et Biophysica Acta (BBA) - Molecular Basis of Disease, vol. 1864, no. 2, pp. 533-541, 2018.

[62] V. Brito, A. Giralt, M. Masana et al., "Cyclin-dependent kinase 5 dysfunction contributes to depressive-like behaviors in Huntington's disease by altering the DARPP-32 phosphorylation status in the nucleus accumbens," Biological Psychiatry, vol. 86, no. 3, pp. 196-207, 2019.

[63] M. Heitzer, S. Kaiser, M. Kanagaratnam et al., "Administration of $17 \beta$-estradiol improves motoneuron survival and down-regulates inflammasome activation in male SOD1(G93A) ALS mice," Molecular Neurobiology, vol. 54, no. 10, pp. 8429-8443, 2017.

[64] Y. x. Ji, M. Zhao, Y. 1. Liu, L. s. Chen, P. l. Hao, and C. Sun, "Expression of aromatase and estrogen receptors in lumbar motoneurons of mice," Neuroscience Letters, vol. 653, pp. 711, 2017.

[65] H. Gong, M. J. Jarzynka, T. J. Cole et al., "Glucocorticoids antagonize estrogens by glucocorticoid receptor-mediated activation of estrogen sulfotransferase," Cancer Research, vol. 68 , no. 18 , pp. 7386-7393, 2008. 